\title{
Taraxacum gilliesii Hook. \& Arn. (Asteraceae), nuevo registro para su área de distribución en Chile
}

\author{
Taraxacum gilliesii Hook. \& Arn. (Asteraceae), \\ new record for its distribution area in Chile
}

Erwin Domínguez ${ }^{1} \&$ Fernán Silva²

En Chile se reconoce la presencia de tres especies del género Taraxacum, dos corresponden a especies nativas: Taraxacum fernandezianum Dahlst. ex Skottsb., herbácea perenne, endémica del archipiélago de Juan Fernández (Marticorena et al. 1998), y Taraxacum gilliesii Hook. \& Arn., hierba perenne, presente en la región de Magallanes (Henríquez et al. 1995; Moore \& Goodall, 1977; Pisano, 1985-86), con una amplia distribución en pastizales naturales áridos a semi-áridos, siendo reportada su presencia en los Parques Nacionales Torres del Paine y Pali Aike (Domínguez et al. 2006; Domínguez et al. 2004). La tercera es Taraxacum officinale F.H. Wigg., una especie introducida, de origen euroasiático, considerada maleza por Matthei (1995) y Espinoza (2017) e invasora de áreas protegidas (Dominguez et al. 2006; Domínguez et al. 2004; Vidal et al. 2015). Ésta fue introducida en la región de Magallanes en 1882 en forma intencional como planta comestible (Moore \& Goodall, 1977). De acuerdo a registros etnobotánicos, los indios ona se alimentaban de plantas del género Taraxacum, aquéllos las denominaban oitá u oitáí (MartínezCrovetto, 1968).

Por otra parte, la única especie citada del género Taraxacum para la región de Aysén del General Carlos Ibáñez del Campo es T. officinale, de acuerdo al Catálogo de las plantas vasculares de Chile (Rodríguez \& Marticorena, 2019). El hallazgo de una población de T. gilliesii formada por $1 \mathrm{a} 3$ individuos por $1.000 \mathrm{~m}^{2}$ (Figs. 1 y 2), creciendo en un coironal de Festuca pallescens, en la Estancia Punta del Monte (4526'55.1"S., $71^{\circ} 34^{\prime} 46.7$ "O.), representa la confirmación de la presencia de esta especie en la región de Aysén, validando una colección anterior realizado en el año
1940 por R. Santesson, la cual fue depositada en el Swedish Museum of Natural History (Uhlemann et al. 2004).

El carácter morfológico más conspicuo de T. gilliesii son las brácteas del involucro, siendo éstas aovadas y erectas a diferencia de T. officinalle, cuyas brácteas son aovado lanceoladas y reflexas (Muiño \& Prina, 2006; Uhlemann et al. 2004).

El material estudiado fue: T. gilliesii Hook. \& Arn.: Chile: región de Aysén, provincia de Coyhaique, comuna de Coyhaique, sector Coyhaique Alto, Estancia Punta del Monte, 29/11/2017, Domínguez \& Silva. MURAY.BH.01 (Museo Regional de Aysén. Botánica Herbario Nro. 01). Con este hallazgo, se amplía su área de distribución en Chile.

\section{AGRADECIMIENTOS}

A la Subsecretaría Ministerial de Agricultura por financiar el programa de investigación de INIA: Sistemas de Praderas Estepáricas de Zonas Frías de Chile, Código: 502093-70.

\section{LITERATURA CITADA}

Domínguez, E., Marticorena, C., Elvebakk, A., y Pauchard, A. (2004). Catálogo de la flora

\footnotetext{
1 Instituto de Investigaciones Agropecuarias (INIA) Casilla 277, Punta Arenas, Chile. 囚edominguez@inia.cl

2 Servicio Agrícola y Ganadero (SAG). Casilla 2690, Coyhaique, Chile.
} 


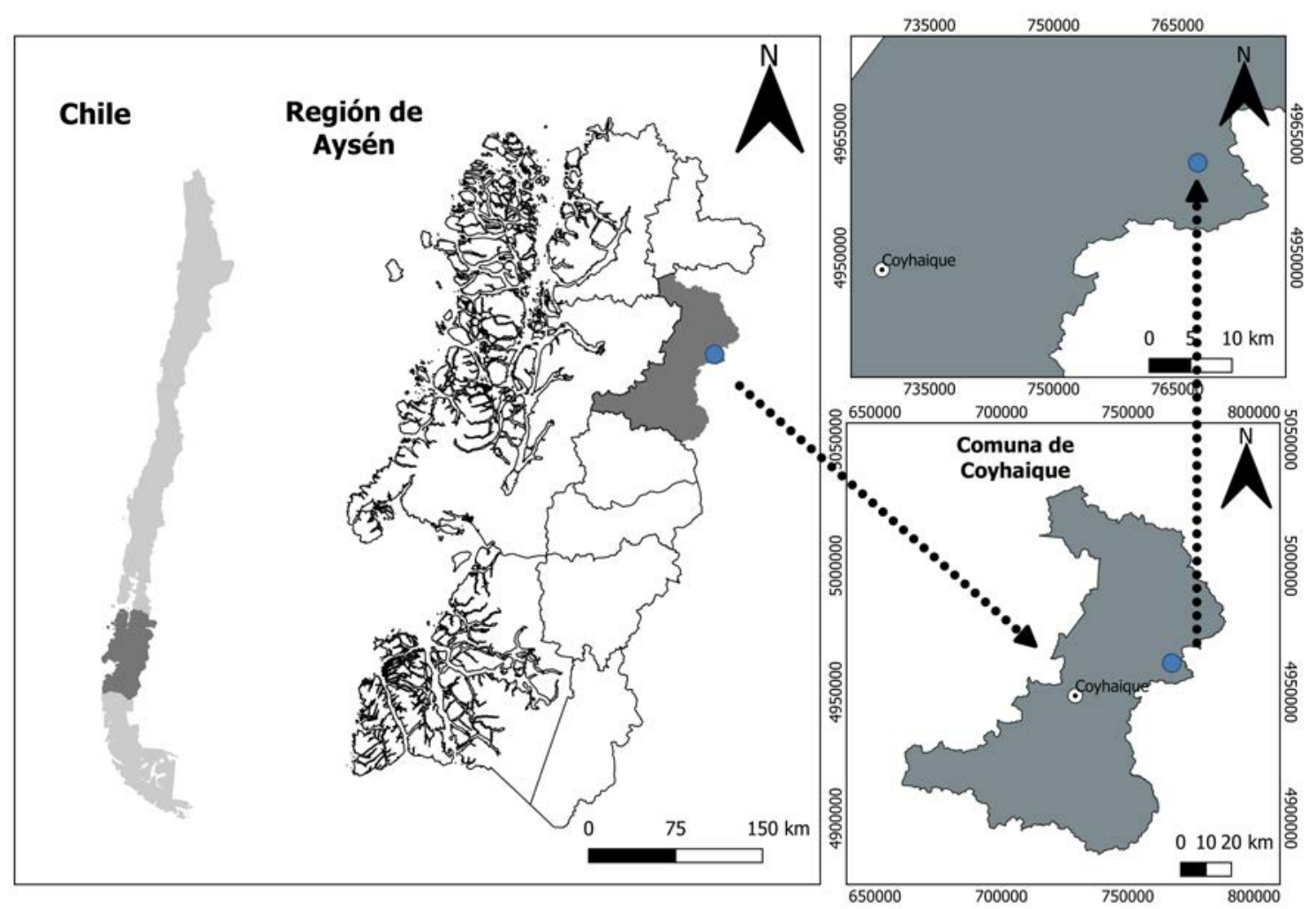

Fig. 1. Localización geográfica de la población de Taraxacum gilliesii Hook. \& Arn., en la comuna de Coyhaique, región de Aysén.

vascular del Parque Nacional Pali Aike. XII Región, Chile. Gayana Botánica, 61(2), 67-72.

Domínguez, E., Elvebakk, A., Marticorena, C., y Pauchard, A. (2006). Plantas introducidas en el Parque Nacional Torres del Paine, Chile. Gayana Botánica, 63(2), 131-141.

Espinoza, N. (2017). Malezas en Chile. Temuco, Chile. Trama Impresores. S.A.

Henríquez, J. M., Pisano, E., y Marticorena, C. (1995). Catálogo de la flora vascular de Magallanes (XII Región), Chile. Anales del Instituto de la Patagonia, 23, 5-30.

Marticorena, C., Stuessy, T. F., y Baeza, C. M. (1998). Catálogo de la Flora vascular de las Islas Robinson Crusoe y Juan Fernández, Chile. Gayana Botñanica, 55(2), 187211.

Martínez-Crovetto, R. (1968). Estudios Etnobotánicos: Nombre de plantas y su utilidad según los indios Onas de Tierra del Fuego. Etnobiológica. Facultad de Agronomía y Veterinaria. Universidad Nacional del Norte. 3, 1-20.
Matthei, O. (1995). Manual de las malezas que crecen en Chile. Santiago: Alfabeta Impresores.

Moore, D. M., y Goodall, R. N. P. (1977). La flora adventicia de Tierra del Fuego. Anales del Instituto de la Patagonia, 8, 263-274.

Muiño, W. A., y Prina, A.O. (2006). Taraxacum gilliesii Hook. \& Arn. (Asteraceae), nuevo registro para su área de distribución en Argentina. Revista Facultad Agronomía UNLPam, 7(2), 51-53.

Pisano, E. (1985-86). Nueva colección referida a Taraxacum rhusiocarpum Dahlst. Anales del Instituto de la Patagonia, 16, 51-53.

Rodríguez, R., y Marticorena, A. (Eds.). (2019). Catálogo de las plantas vasculares de Chile. Editorial Universidad de Concepción.

Uhlemann, I., Kirschner, J., y Štěpánek, J. (2004). The genus Taraxacum (Asteraceae) in the Southern hemisphere. I. The section Antarctica Handel-Mazzetti and notes on dandelions of Australasia. Folia Geobotanica, 39, 205-220. 

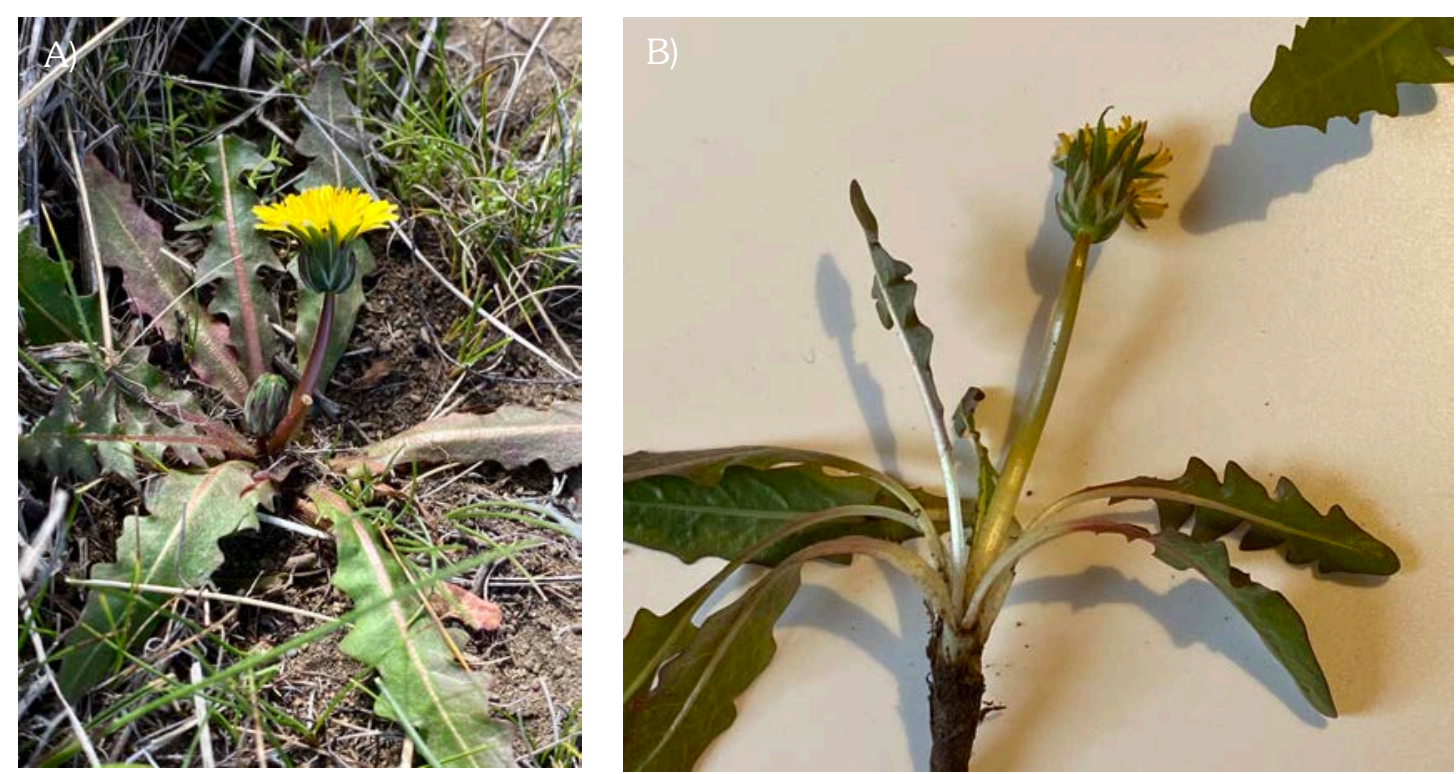

Fig. 2. Taraxacum gilliesii en la región de Aysén: A) en su hábitat natural y

B) ejemplar herborizado depositado en el Museo Regional de Aysén.

Vidal, O. J., Aguayo, M., Niculcar, R., Bahamonde, N., Radic, S., San Martín, C., Kusch, A., Latorre, J., y Félez, J. (2015). Plantas invasoras en el Parque Nacional Torres del Paine (Magallanes, Chile): Estado del arte, distribución post-fuego e implicancias en restauración ecológica. Anales del Instituto de la Patagonia, 43, 75-96. 